\title{
Insights into the segmental identity of post-oral commissures and pharyngeal nerves in Onychophora based on retrograde fills
}

\author{
Christine Martin ${ }^{1,2^{*}}$ and Georg Mayer ${ }^{1,2}$
}

\begin{abstract}
Background: While the tripartite brain of arthropods is believed to have evolved by a fusion of initially separate ganglia, the evolutionary origin of the bipartite brain of onychophorans — one of the closest arthropod relatives — remains obscure. Clarifying the segmental identity of post-oral commissures and pharyngeal nerves might provide useful insights into the evolution of the onychophoran brain. We therefore performed retrograde fills of these commissures and nerves in the onychophoran Euperipatoides rowelli.
\end{abstract}

Results: Our fills of the anterior and posterior pharyngeal nerves revealed groups of somata that are mainly associated with the deutocerebrum. This resembles the innervation pattern of other feeding structures in Onychophora, including the jaws and several lip papillae surrounding the mouth. Our fills of post-oral commissures in E. rowelli revealed a graded arrangement of anteriorly shifted somata associated with post-oral commissures \#1 to \#5. The number of deutocerebral somata associated with each commissure decreases posteriorly, i.e., commissure \#1 shows the highest and commissure \#5 the lowest numbers of associated somata, whereas none of the subsequent median commissures, beginning with commissure \#6, shows somata located in the deutocerebrum.

Conclusions: Based on the graded and shifted arrangement of somata associated with the anteriormost post-oral commissures, we suggest that the onychophoran brain, which is a bipartite syncerebrum, might have evolved by a successive anterior/anterodorsal migration of neurons towards the protocerebrum in the last onychophoran ancestor. This implies that the composite brain of onychophorans and the compound brain of arthropods might have independent evolutionary origins, as in contrast to arthropods the onychophoran syncerebrum is unlikely to have evolved by a fusion of initially separate ganglia.

Keywords: Central nervous system, Brain, Pharynx, Neuronal tracing, Arthropod, Panarthropod

\section{Background}

The typical arthropod brain is composed of three segmental regions: the protocerebrum, deutocerebrum, and tritocerebrum [1-6]. In contrast, the brain of one of the closest arthropod relatives, the Onychophora (velvet worms), contains only two segmental regions corresponding to the arthropod proto- and deutocerebrum $[7,8]$. Alternative hypotheses suppose a tripartite onychophoran brain [9] but are based on a misinterpretation of

\footnotetext{
*Correspondence: christine.martin@uni-leipzig.de

1 Animal Evolution and Development, Institute of Biology, University

of Leipzig, Talstraße 33, 04103 Leipzig, Germany

Full list of author information is available at the end of the article
}

the onychophoran neuroanatomy, as the authors mistook the jaw nerve for the slime papilla nerve. Furthermore, a recent study [10] proposes that the onychophoran brain is a monopartite structure based on engrailed mRNA expression [11, 12], a method that is not suitable for addressing brain segmentation, as the anterior engrailed stripe is on the non-neuroectodermal side [13]. Despite the lack of a distinct border delineating these two brain regions, the protocerebrum of onychophorans contains the central body and the mushroom bodies and innervates the antennae and eyes, whereas the deutocerebrum is defined by the position of neuronal somata supplying the appendages of the second body segment, i.e., the jaws 
$[7,8,14]$ (Fig. 1a). The onychophoran jaws are the only appendages that become incorporated into the definitive mouth cavity during embryonic development [14, 15] (Fig. 1b-e). In contrast to the antennae and jaws, the third pair of cephalic appendages of onychophorans, the slime papillae, is not innervated by the brain but rather by the anterior portions of the ventral nerve cords $[7,8$, 16] (Fig. 1a).

The two widely separated ventral nerve cords of onychophorans do not show any segmental ganglia but instead are linked with each other by numerous median and ring commissures in an orthogon-like fashion [16-19]. The nerve cords are connected to the brain via a pair of medullary (i.e. non-ganglionated) structures, the so-called connecting cords [7] ("circumoesophageal connectives" sensu Henry [20]; "medullary connectives" sensu Whitington and Mayer [16] "connecting pieces" sensu Martin and Mayer [14]). The frequently used term "circumoesophageal connectives" is unsuitable for designating these cords, as connectives are somata-free structures connecting ganglia [5], whereas the connecting cords resemble the ventral nerve cords. Nevertheless, the connecting cords differ from the ventral nerve cords in that they are not associated with ring commissures. The connecting cords are situated on each side of the pharynx and linked with each other via a number of post-oral (=post-pharyngeal) commissures. Due to the medullary organization of the connecting cords, their segmental identity as well as that of the associated commissures is unclear.

In addition to the post-oral commissures, Henry [20] described a pair of "stomodaeal nerves" emanating from the connecting cords and supplying the pharynx. As with the post-oral commissures associated with the connecting cords, the segmental identity of this pair of pharyngeal nerves is unknown because the corresponding neuronal somata have not been identified. An additional prominent "loop nerve" is also associated with the onychophoran pharynx [4]. Although this nerve shows a medullary organization, with at least some somata located in the dorsolateral pharyngeal wall, it is unclear whether or not there are additional neurons supplying this nerve located within the brain. Clarifying this issue would help to determine the innervation pattern and segmental identity of the onychophoran pharynx, which remains ambiguous.

During ontogeny, the onychophoran pharynx arises from the walls of the embryonic stomodeum [15, 21]. This would suggest that the pharynx, like the stomodeum itself, belongs to the second body segment. Accordingly, the first post-oral commissure of onychophorans, which
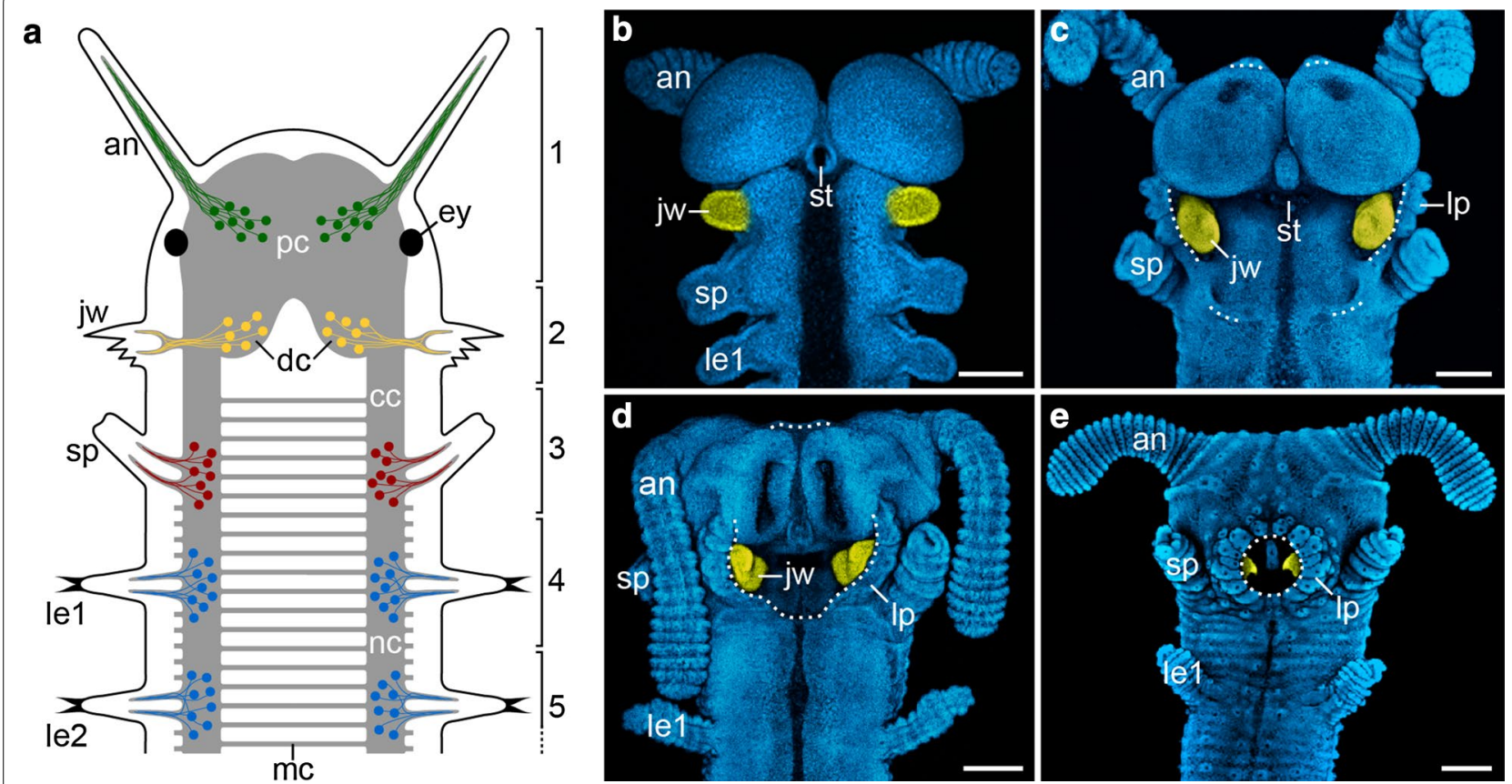

Fig. 1 Segmental identity of cephalic appendages and head development in onychophorans. a Simplified diagram of the innervation of segmental cephalic appendages. Body segments, each carrying a pair of appendages, are numbered. b-e Head development in embryos of subsequent developmental stages in the onychophoran Euperipatoides rowelli. The jaws are highlighted artificially in yellow. Modified from Ou et al. [15]. an antenna, cc connecting cord, dc deutocerebrum, ey eye, jw jaw, lel and le2 first and second legs, Ip lip papillae, mc median commissure, nc nerve cord, pc protocerebrum, st stomodeum, sp slime papilla. Scale bars (in b-e) $200 \mu \mathrm{m}$ 
is closely associated with the ventral pharyngeal wall [20], might also belong to the second body segment. Alternatively, this commissure might be composed of fibers from both the second (jaw) and the third (slime papilla) segments, thus resembling the situation in arthropods, in which the first post-oral commissure contains both deutocerebral and tritocerebral fibers [1, 22-26].

To clarify the composition of post-oral commissures and the segmental identity of pharyngeal nerves in Onychophora, we performed retrograde fills of these neural structures and localized the position of their supplying neurons in the onychophoran Euperipatoides rowelli.

\section{Results}

\section{Arrangement and innervation pattern of the anterior post-oral commissures}

Dissected portions of the anterior nervous system of $E$. rowelli show that the arrangement of the five anteriormost median commissures differs from that of the remaining commissures connecting the two nerve cords of the trunk (Fig. 2a, b). The first commissure links the two ring commissure-free connecting cords at a distance of about one-third between the slime papilla and the jaw nerves. This prominent commissure is longer and thicker than the remaining post-oral commissures, from which it is segregated by a wider gap. The first post-oral commissure forms a ventral loop around the pharynx and is attached to the ventral pharyngeal wall by thin tissue fibers so that this commissure usually has to be dissected from the pharynx during preparation. The commissures \#2 to \#5 link the nerve cords at the level of the third lip papillae nerve and the slime papillae nerves (Fig. 2a, b). These commissures lie closer to each other than do the remaining median commissures of the trunk.

To determine the position of neuronal somata associated with the anterior post-oral commissures, we performed retrograde fills of commissures \#1 to \#5 and compared their innervation pattern to that of the median commissures from the remaining trunk. Our bidirectional fills revealed eight groups of somata associated with the first post-oral commissure, i.e., four groups labeled by each fill (Fig. 3a-d). Three of the four groups are located dorsally within the deutocerebrum:

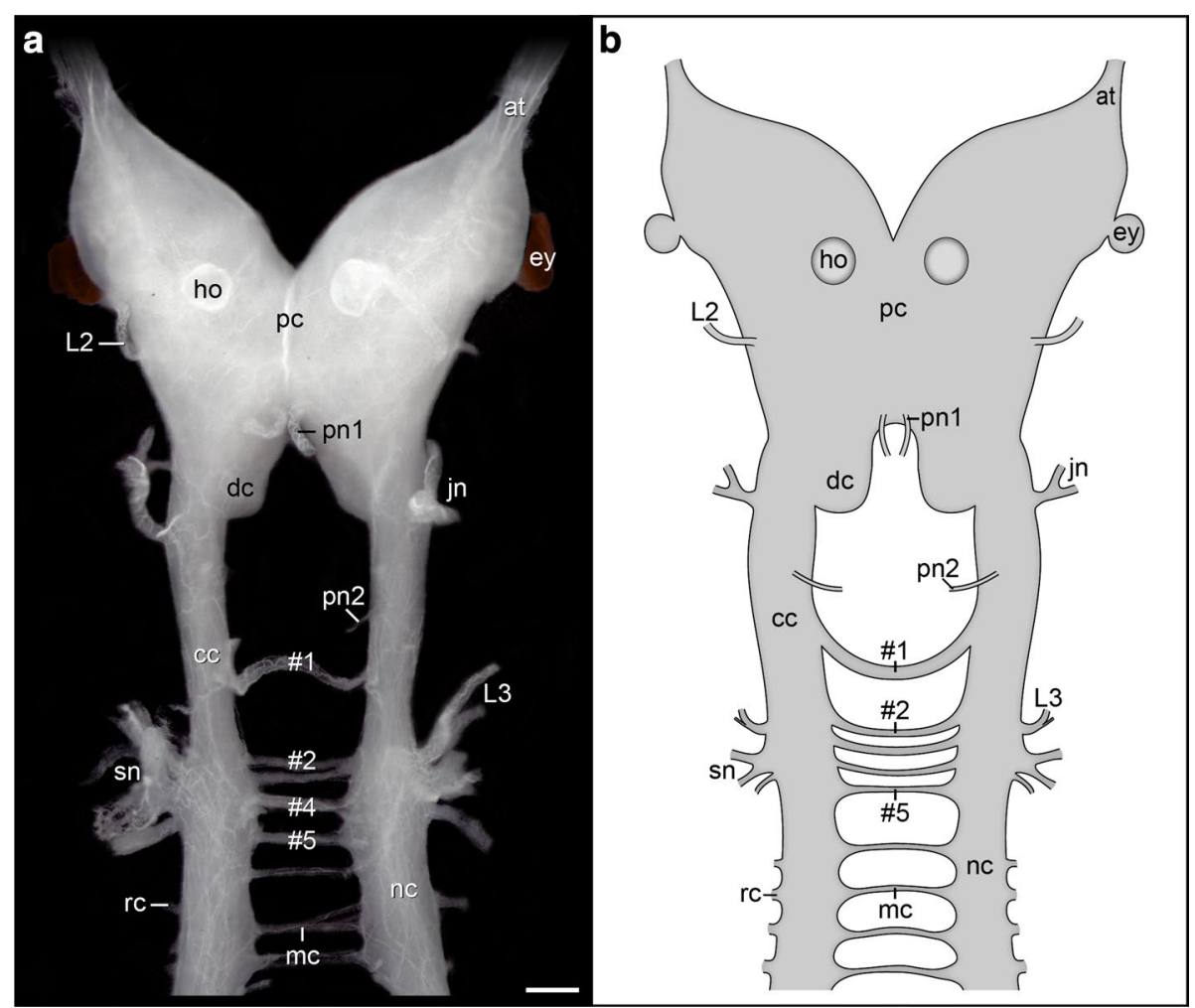

Fig. 2 Anatomy of the anterior nervous system in the onychophoran Euperipatoides rowelli. Light micrograph (a) and simplified diagram (b) of dissected brain and anterior nerve cords in ventral view. Anterior is up. Anteriormost post-oral commissures numbered (\#1 to \#5). at antennal tract, cc connecting cord (characterized by the absence of ring commissures), dc deutocerebrum, ey eye, ho hypocerebral organ, jn jaw nerve, L2 and L3 second and third lip papillae nerves; mc, median commissure, $n c$ nerve cord, pc protocerebrum, pn 1 and pn 2 anterior and posterior pharyngeal nerves, rc ring commissure, sn slime papilla nerves. Scale bar (in a) $200 \mu \mathrm{m}$ 


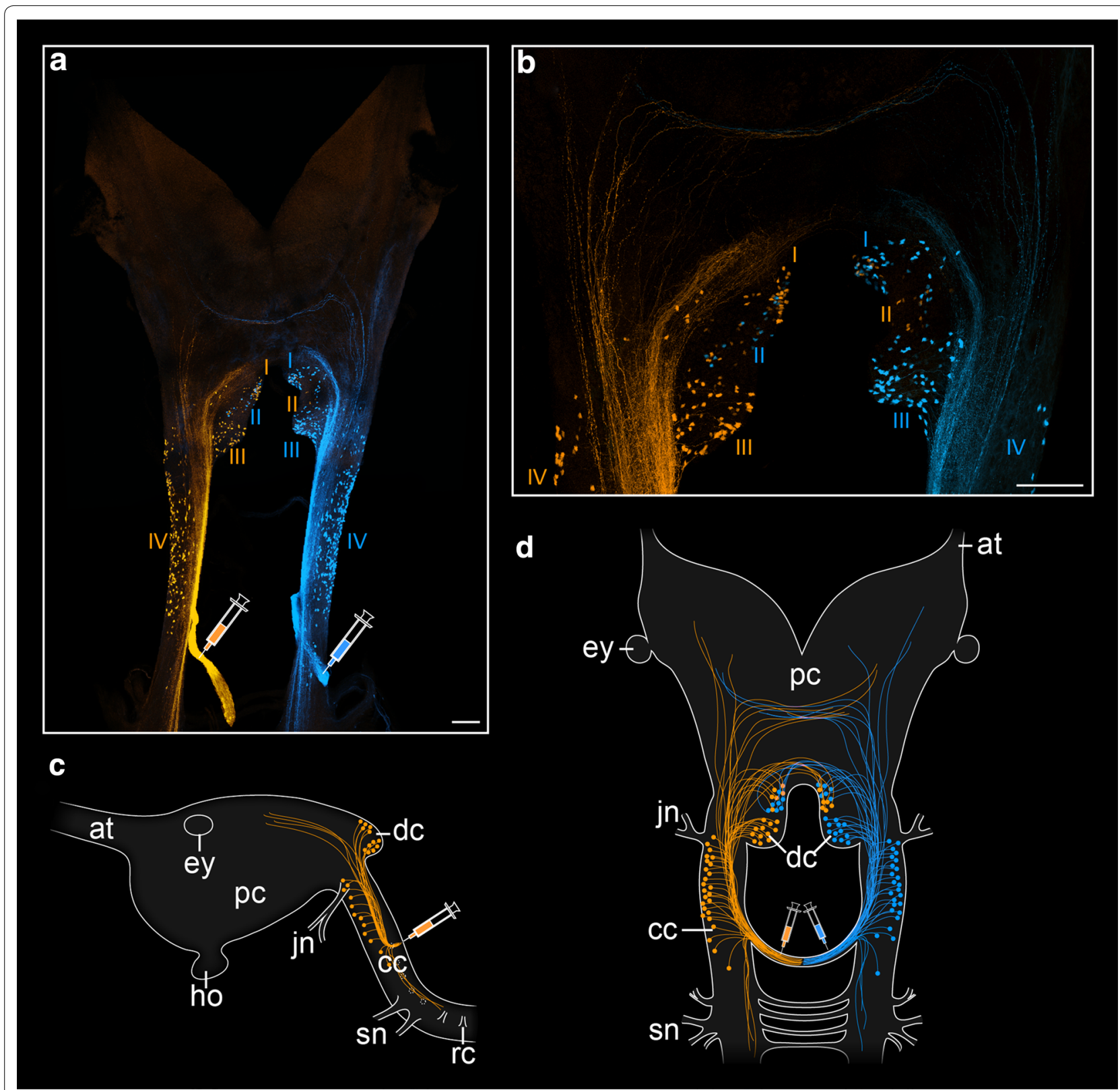

Fig. 3 Localization of neuronal somata associated with the first post-oral commissure in Euperipatoides rowelli. Retrograde fills. Syringes indicate the corresponding fill sites. a, b Maximum projection confocal micrograph of a double fill with dextran coupled to two different fluorochromes. Dorsal view, anterior is up. Note the position of somata in the connecting cords (group IV) and in the deutocerebrum (groups I-III), some of which lie in the contralateral brain hemisphere (group II). c Overview diagram illustrating the position of neurons associated with the first post-oral commissure in lateral view. d Overview diagram demonstrating the position of neurons associated with the first post-oral commissure in dorsal view. at antennal tract, $c c$ connecting cord, $d c$ deutocerebrum, ey eye, ho hypocerebral organ, jn jaw nerve, $p c$ protocerebrum, rc ring commissure, sn slime papilla nerves. Scale bars (in a, b) $100 \mu \mathrm{m}$

two (with $\sim 30$ and $\sim 50$ somata each) in the ipsilateral half and one (with $\sim 20$ somata) in the contralateral half of the deutocerebrum, with respect to the site of the fill. The fourth and largest group of 100 somata is located laterally in each connecting cord. The somata of this group are distributed along each connecting cord from the basis of the first post-oral commissure to the basis of the jaw nerve (Fig. 3a, c, d). In addition to the neuronal somata, our fills of the first post-oral commissure revealed numerous anterior and posterior fibers, which terminate within both the protocerebrum and the nerve cords (Fig. 3a-d). 


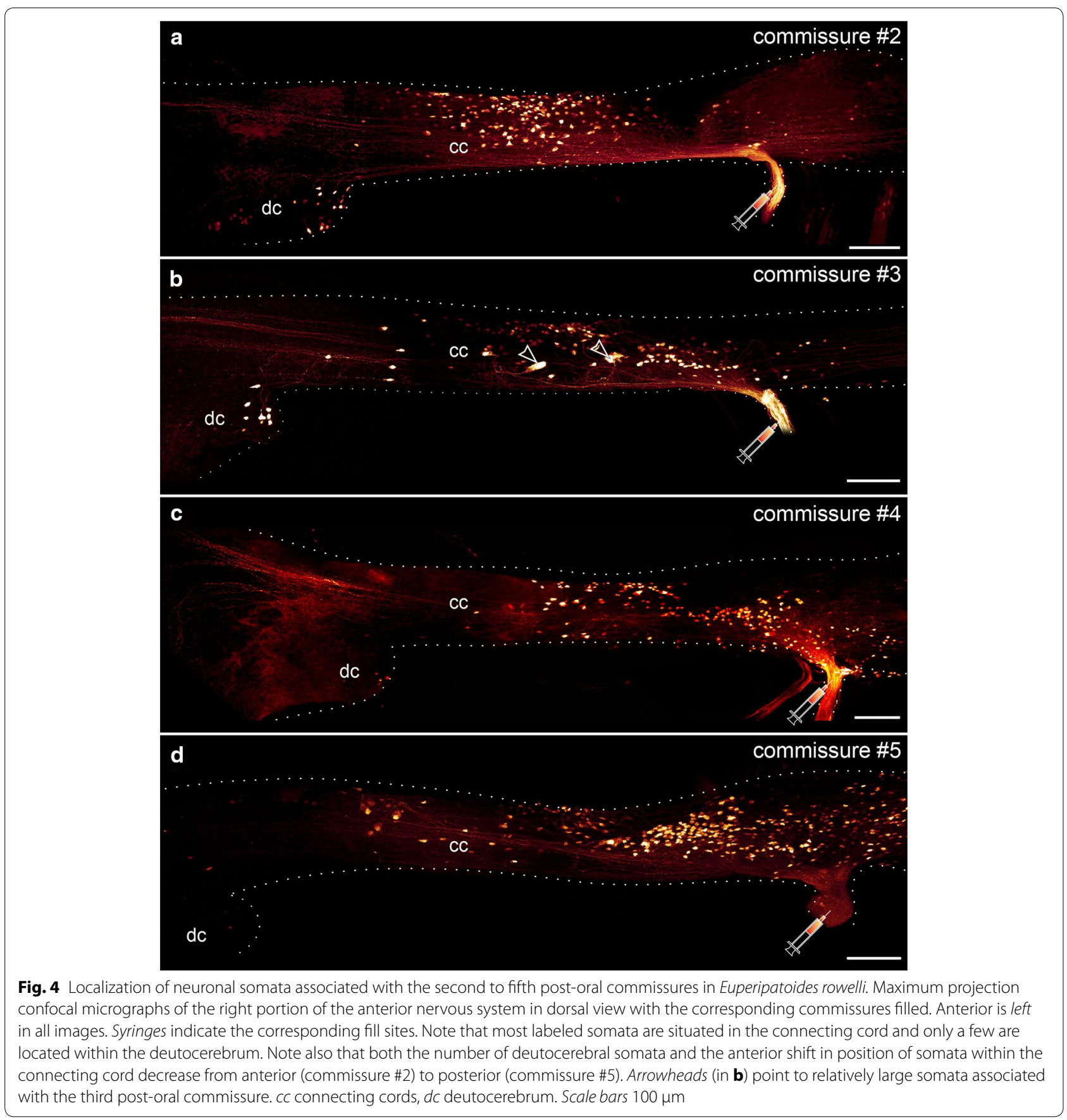

In contrast to the first post-oral commissure, commissures \#2 to \#5 do not show any contralateral clusters of somata within the brain with respect to the site of the fill (Fig. 4a-d). All somata are instead located in the ipsilateral half of the nervous system, including the deutocerebrum and the connecting cord. The number of somata located within the deutocerebrum decreases from commissure \#2 to commissure \#5. The somata associated with each commissure exhibit an anterior shift in the connecting cord with respect to the basis of the corresponding commissure. This shift is more prominent for the anteriormost commissures and less evident for those situated further posteriorly (Figs. 3a, $4 a-d)$. In contrast to the first post-oral commissure, the somata associated with commissures \#2 to \#5 are not located laterally in the connecting cord but 
instead occupy most of its width. Most filled somata associated with post-oral commissures \#2 to \#5 are uniform in size $(5-10 \mu \mathrm{m}$ in diameter), but a few relatively large somata $(25-30 \mu \mathrm{m}$ in diameter) are associated with commissure \#3 (arrowheads in Fig. 4b). Like the first post-oral commissure, commissures \#2 to \#5 exhibit additional anterior fibers that project into the protocerebrum.

While a slight anterior shift in the position of somata associated with commissures \#2 to \#5 is evident within the connecting cords, the remaining median commissures of the trunk, beginning with commissure \#6, do not show any such shift. All somata associated with these commissures are instead grouped at the level of each commissure (Fig. 5a, b).

\section{Neuronal tracing of nerves supplying the pharynx}

Of the two pairs of nerves supplying the pharynx in $E$. rowelli, the anterior pair enters the brain ventrally, where it is associated with the anterior portion of the deutocerebrum (Figs. 2a, b, 6a). In contrast, the posterior pair of pharyngeal nerves is associated with the portions of the connecting cords anterior to the first post-oral commissure (Figs. 2a, b, 6b). Retrograde fills of the anterior pharyngeal nerve revealed $\sim 60$ somata located exclusively within the deutocerebrum in the same brain hemisphere, posterior to the basis of this nerve (Fig. 6a). There are two major groups of somata: those of group I (Fig. 6a) project their axons exclusively anteriorly, whereas the axons of group II (Fig. 6a) leave the somata posteriorly and are then reoriented anteriorly. In addition, there are a few somata that are located further anteriorly within the deutocerebrum (Fig. 6a). Some of the fibers associated with the anterior pharyngeal nerve extend further anteriorly in the protocerebrum as well as in the contralateral brain hemisphere, where they terminate.

Like the anterior pair of nerves supplying the pharynx, the posterior pair also shows neuronal somata located within the deutocerebrum (Fig. 6b). However, apart from these, each posterior pharyngeal nerve has a few additional somata $(\sim 10)$ in each connecting cord that are grouped around the basis of the nerve (Fig. 6b). The
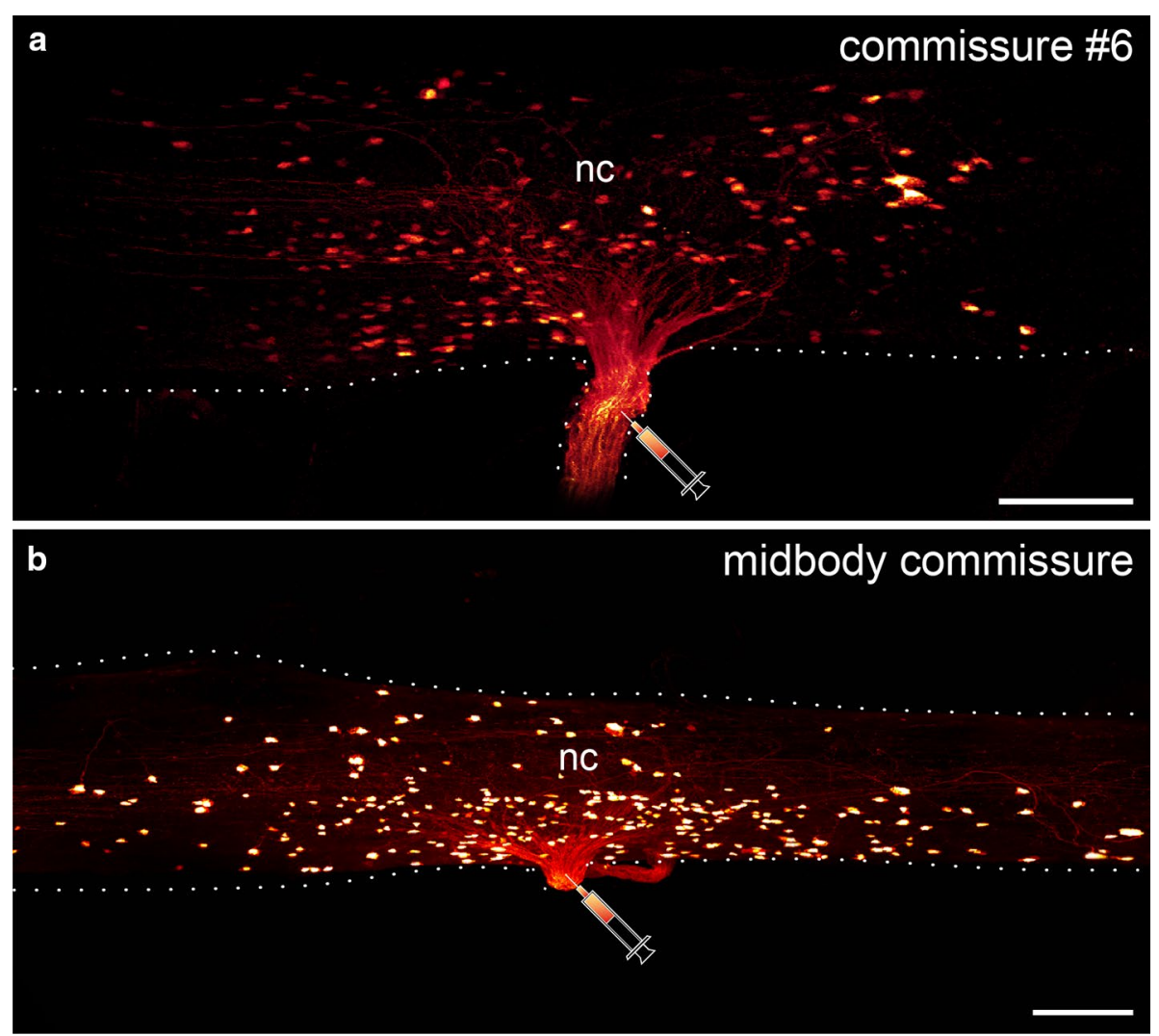

Fig. 5 Localization of neuronal somata associated with the sixth post-oral and a midbody commissure in Euperipatoides rowelli. Retrograde fills. a, $\mathbf{b}$ Maximum projection confocal micrographs. Anterior is left in both images. Syringes indicate the corresponding fill sites. Note the similar arrangement of neuronal somata that are grouped near the basis of each commissure and do not show any anterior shift in position. nc nerve cord. Scale bars $100 \mu \mathrm{m}$ 

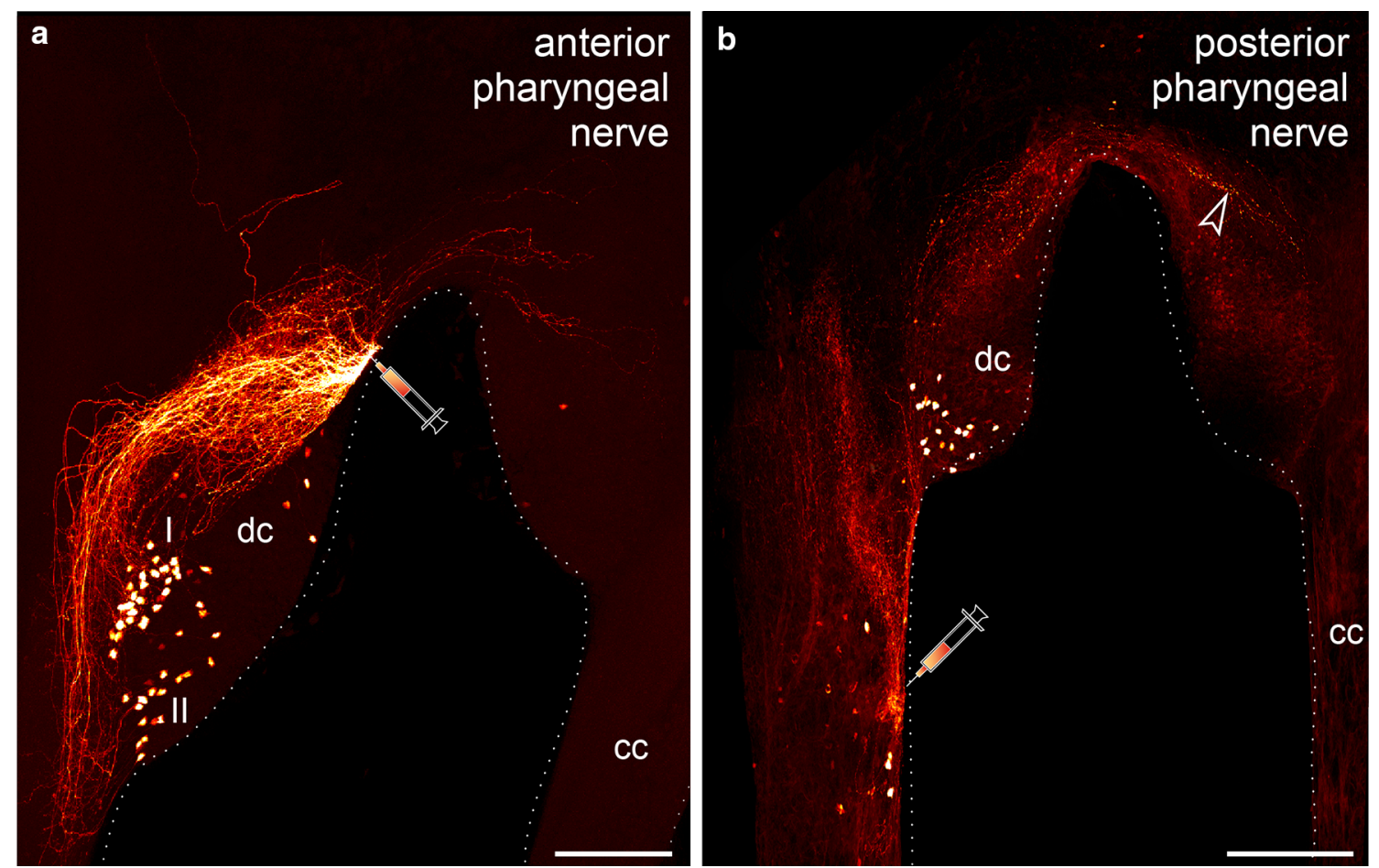

Fig. 6 Localization of neuronal somata associated with the anterior and posterior pharyngeal nerves in Euperipatoides rowelli. Retrograde fills. Maximum projection confocal micrographs in dorsal view. Anterior is up. Syringes indicate the corresponding fill sites. a Fill of the first pharyngeal nerve showing two groups of somata (I and II) associated with the deutocerebrum. b Fill of the second pharyngeal nerve illustrating neuronal somata within the deutocerebrum as well as in the connecting cord. Arrowhead points to fibers terminating contralaterally. cc connecting cord, $d c$ deutocerebrum. Scale bars $100 \mu \mathrm{m}$

group of somata within the deutocerebrum comprises $\sim 30$ cells that are all situated in the ipsilateral brain hemisphere. A number of fibers associated with the posterior pharyngeal nerve terminate in the connecting cord as well as in the contralateral half of the brain (arrowhead in Fig. 6b).

\section{Discussion}

\section{Deutocerebral innervation of feeding structures \\ in Onychophora}

Our retrograde fills of the anterior and posterior pairs of pharyngeal nerves in E. rowelli revealed that the majority of neuronal somata supplying the onychophoran pharynx are associated with the deutocerebrum (Fig. 7a, b). This innervation pattern of the adult pharynx corresponds to the embryonic origin of this structure from the walls of the stomodeum, which arise from the invaginating ectoderm near the border between the first and second body segments [15, 21] (cf. Fig. 1b). Since the stomodeum arises posterior to the hedgehog domain of the antennal segment but anterior to that of the jaw segment [12], it can be unambiguously assigned to the second, i.e., deutocerebral body segment. Thus, the segmental identity of the pharynx and its anlage as a deutocerebral structure is supported by both previous developmental and our neuroanatomical data. Besides the pharynx, additional structures involved in feeding that are innervated by the deutocerebrum are the jaws and several lateral or posterolateral lip papillae surrounding the mouth $[8,14]$. This suggests that the deutocerebrum plays a role in coordinating feeding structures in Onychophora.

\section{Segmental identity of post-oral commissures in Onychophora}

The median commissures that link the two widely separated nerve cords of onychophorans do not show any obvious segmental arrangement but are instead organized in a ladder-like fashion along the body (reviewed by Whitington and Mayer [16] and Mayer [7]). Using the position of leg nerves as segmental landmarks, previous studies have shown that the number of median commissures varies between 8 and 10 per segment [16-19, 27]. This variation in number and the lack of segmental ganglia in onychophorans generally render it difficult to assign each median commissure to a corresponding body segment.

Our fills of trunk commissures revealed that their supplying neurons are grouped around each commissural basis. 


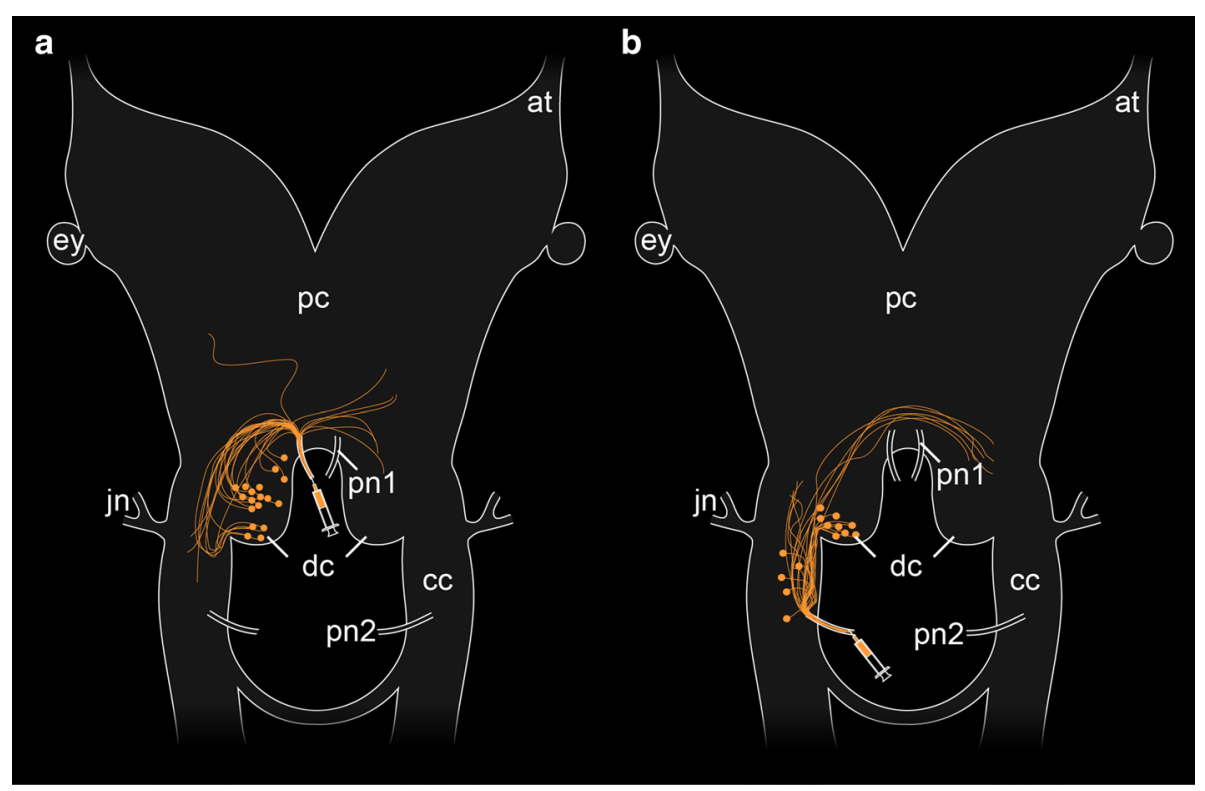

Fig. 7 Simplified diagrams summarizing the results of retrograde fills of the pharyngeal nerves in Euperipatoides rowelli. Anterior is up. Syringes point to the corresponding fill sites. Note the somata associated with the anterior and posterior pharyngeal nerves that are situated within the deutocerebrum $(\mathbf{a}, \mathbf{b})$ and additional somata associated with the posterior pharyngeal nerve that are located in the connecting cord (b). at antennal tract, $c c$ connecting cord, $d c$ deutocerebrum, ey eye, jn jaw nerve, $p c$ protocerebrum, $p n 1$ anterior pharyngeal nerve, pn2 posterior pharyngeal nerve

This holds true for all median commissures of the trunk, including commissure \#6 (Fig. 8f). In contrast, post-oral commissures \#1 to \#5 display numerous anteriorly shifted somata, some of which clearly lie within the deutocerebrum (Fig. 8a-e). The anteriorly shifted position of somata makes it difficult to assign each post-oral commissure to a particular head segment. However, the position of somata within the deutocerebrum as well as in portions of nerve cords supplying the slime papillae suggests that commissures \#2 to \#5 receive fibers from both the second (jaw) and the third (slime papilla) segments (Fig. 8b-e). This pattern resembles the innervation of the first post-oral commissure in various arthropods, which contains both deutocerebral and tritocerebral fibers [1, 22-26] (Fig. 9a, b).

Our neuroanatomical data from $E$. rowelli further revealed that the first post-oral commissure differs from commissures \#2 to \#5 in that it is thicker and longer and lies relatively further anteriorly, as it is associated with the connecting cords rather than with the anterior portions of nerve cords supplying the slime papillae (cf. Fig. 2a, b). Moreover, the first post-oral commissure is the only commissure with somata in the contralateral brain hemispheres relative to the fill site (Figs. 3b, 8a). Despite our detailed data on the position of supplying neurons, the first post-oral commissure of onychophorans is difficult to assign to a particular head segment, as numerous associated somata are located in the connecting cords, the segmental identity of which is unknown.
However, since most commissural somata are situated in the anterior portions of the connecting cords as well as in the deutocerebrum and none of them is associated with the regions of nerve cords innervating the slime papillae, we suggest that the first post-oral commissure mainly, if not exclusively, receives fibers from neurons that belong to the second (jaw) segment (Fig. 9b). Clarifying the segmental identity and embryonic origin of the connecting cords will be crucial for substantiating this hypothesis.

In summary, our findings suggest that the first postoral commissure of onychophorans is most likely associated with the second (deutocerebral) part of the brain supplying the jaws [8]. Interestingly, Henry [20] reported a close association of the first post-oral commissure with the ventral pharyngeal wall, which was also evident in our preparations of the nervous system in E. rowelli. We therefore cannot exclude that at least some commissural fibers might project into the pharynx. If true, this would imply that the first post-oral commissure is involved in the control of pharyngeal function, i.e., it might play a role in controlling the ingestion of food.

\section{Conclusion: implications for the evolution of the onychophoran brain}

The overall organization of the onychophoran brain differs from that of arthropods in that it has only two segmental regions, the protocerebrum and the deutocerebrum [8] (Fig. 1a). In contrast, representatives 


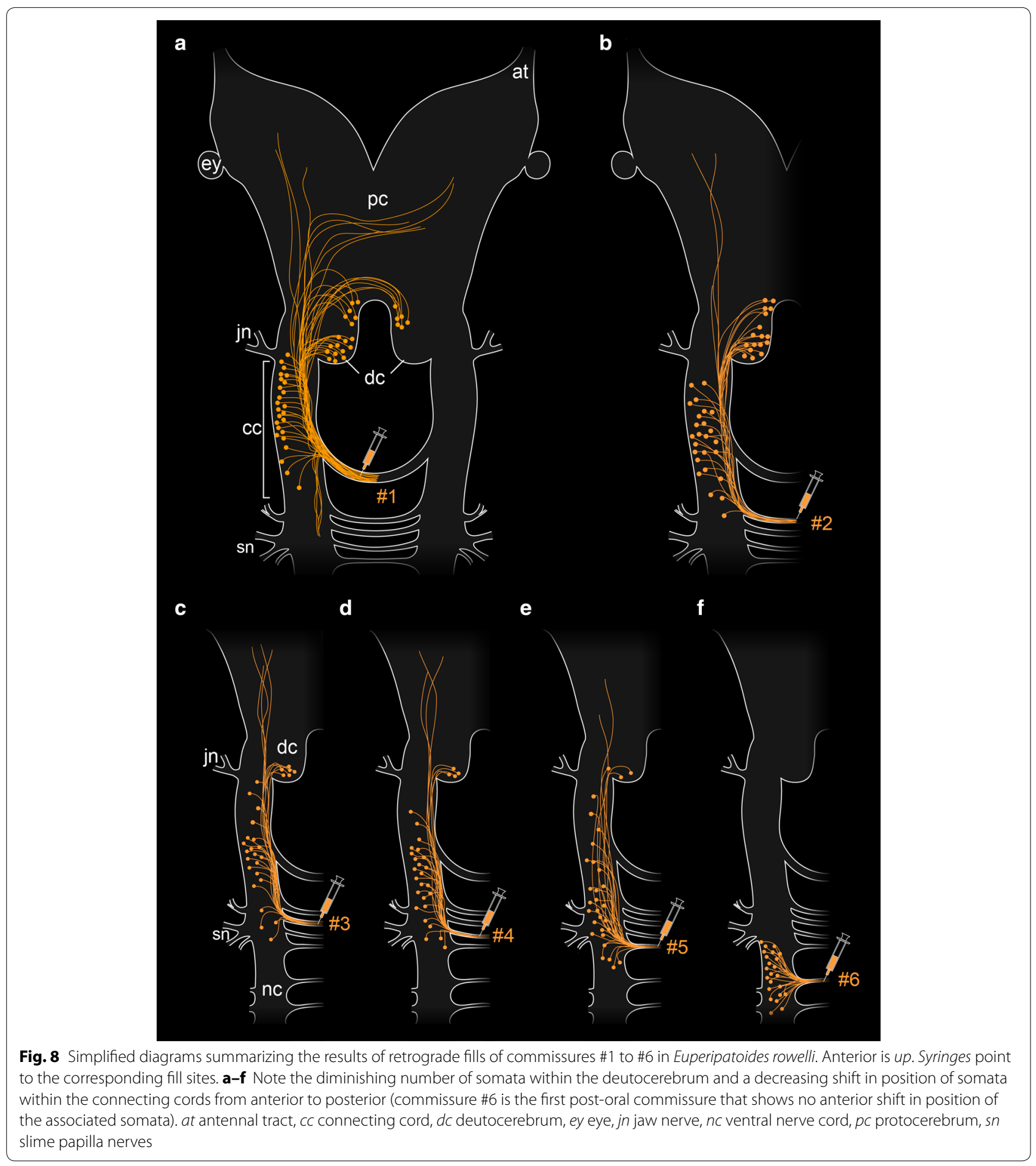

of arthropods typically have at least one additional segmental brain region, the tritocerebrum (e.g. $[1-3,6$, 28]; but note also that branchiopod crustaceans have a bipartite brain since the tritocerebrum is spatially separated from the proto- and deutocerebrum [29]). The typical arthropod brain is a compound structure, which is formed by the morphological fusion of separate embryonic ganglion anlagen [5]. Thus, the ontogeny of the arthropod brain might recapitulate evolutionary changes that have taken place in the arthropod lineage, as the 


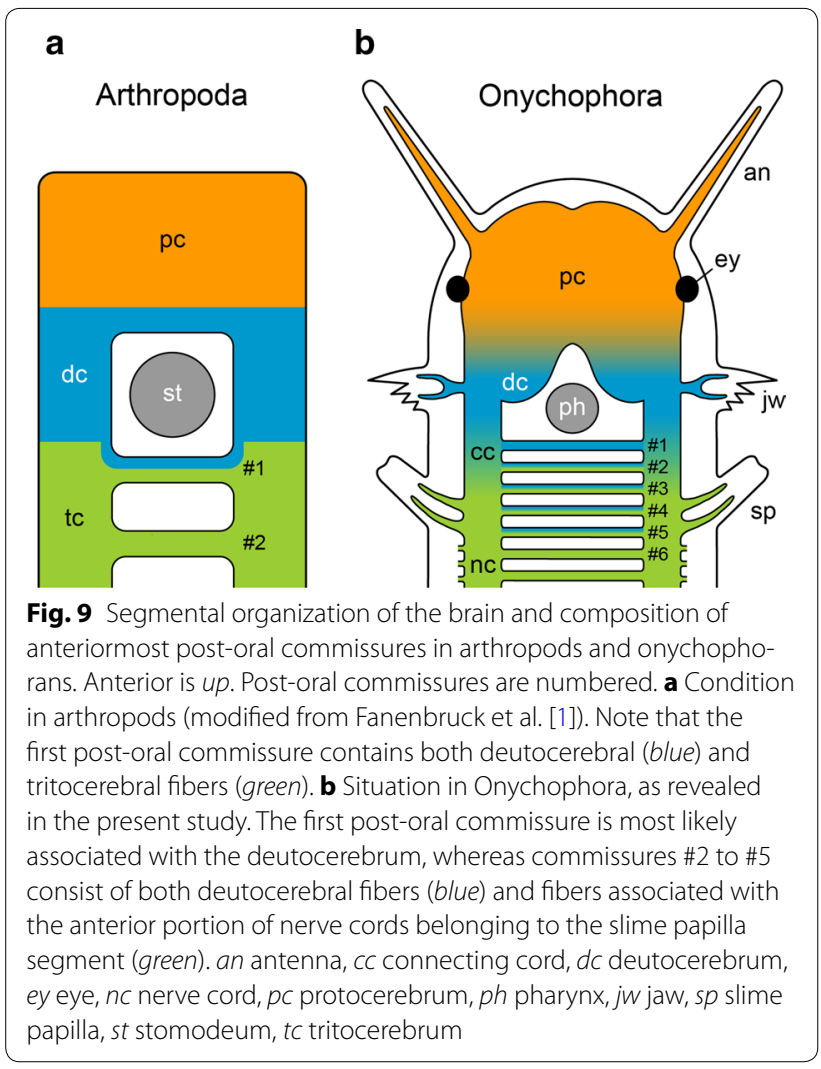

tripartite brain of arthropods might have evolved from separate ganglia that have fused to form a syncerebrum [5] (Additional file 1). A segmentally ganglionated nervous system is also present in tardigrades [30-34], one of the closest relatives of arthropods. However, developmental studies [35] argue against a multisegmented brain in tardigrades. Additionally, the position of the stomatogastric ganglion in the second trunk segment [4] suggests that this segment in tardigrades is homologous to the arthropod tritocerebrum. Accordingly, the ganglion of the first trunk segment corresponds to the arthropod deutocerebrum and therefore the tardigrade brain consists of only a single segmental region corresponding to the arthropod protocerebrum. Thus, Tardigrada might represent the ancestral "rope ladder-like" condition, i.e. one ganglion per segment, suggesting that ganglionization occurred before the fusion of brain neuromeres, as is found in arthropods. Such a ganglionic fusion is unlikely to have occurred in onychophorans because their last common ancestor most likely did not possess any segmental ganglia and because no segmental ganglion anlagen exist in the onychophoran embryo [17-19]. Hence, the bipartite brain of onychophorans might have evolved by an entirely different process.

Our retrograde fills revealed a graded arrangement of anteriorly shifted somata associated with post-oral commissures \#1 to \#5 in E. rowelli (cf. Fig. 8a-e). The number of deutocerebral somata associated with each commissure decreases posteriorly, i.e., commissure \#1 shows the highest and commissure \#5 the lowest numbers, whereas none of the subsequent commissures, beginning with commissure \#6, exhibits somata located in the deutocerebrum. Based on this graded arrangement of commissural neurons, we assume that the deutocerebral somata might have migrated anteriorly along each nerve cord, i.e., towards the protocerebrum, in the onychophoran lineage (Additional file 2). The medullary organization of the ventral nervous system in onychophorans $[7,16-19,36]$ might have allowed the neuronal somata to change their position along the antero-posterior body axis in their last common ancestor. This hypothesis does not require an assumption of pre-existing segmental ganglia in the onychophoran ancestor that might have fused to a compound brain, as in arthropods.

These findings imply that the bipartite syncerebrum of onychophorans and the tripartite brain of arthropods might have evolved convergently by two different processes: an anterior migration of neurons in the onychophoran lineage, and a fusion of pre-existing segmental ganglia in the arthropod lineage (Additional files 1, 2). To distinguish between the two types of brain, Richter et al. [5] suggested the terms "compound" for the arthropod brain and "composite" for the onychophoran brain-a suggestion that receives support from our observations.

Within the group comprising onychophorans, tardigrades and arthropods (panarthropods) the position of tardigrades and onychophorans is still unresolved [31, 37-40]. Onychophorans are regarded as the sister group to either arthropods (Fig. 10a) or arthropods and tardigrades (Fig. 10b). Assuming that tardigrades form the sister group of onychophorans and arthropods (Fig. 10a), the last common ancestor of panarthropods might have possessed separate segmental ganglia and a monopartite brain. After a fusion of the two anteriormost ganglia to a bipartite brain in the last common ancestor of onychophorans and arthropods, the remaining segmental ganglia were lost within the onychophoran lineage, whereas most arthropods evolved a tripartite brain due to an additional fusion (Fig. 10a). Consequently, this scenario assumes that both onychophorans and arthropods possess a compound brain that evolved in their last common ancestor.

Alternatively, assuming that Onychophora form the sister group to tardigrades and arthropods, the last common ancestor of panarthropods might have possessed a monopartite brain and medullary ventral nerve cords (Fig. 10b). Within the onychophoran lineage, neurons of the second body segment migrated towards the 
a

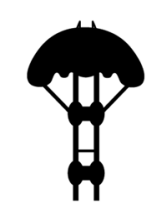

Tardigrada

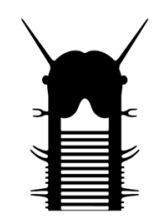

nychophora

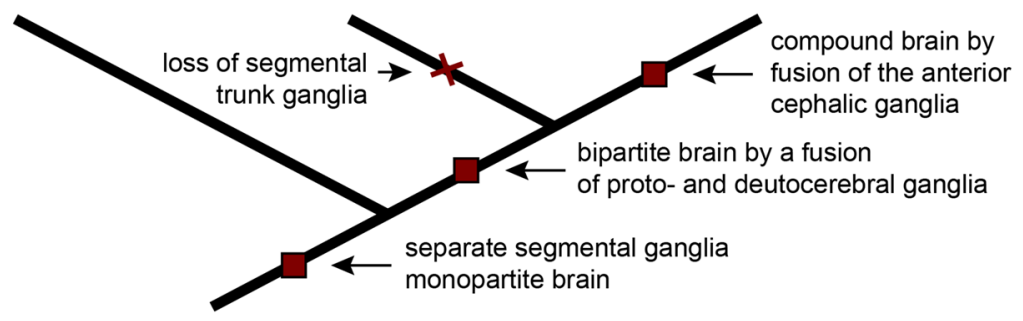

b
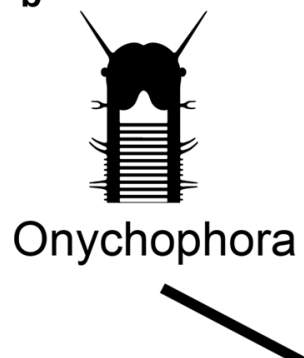

composite (bipartite) brain by anterior migration of deutocerebral somata
Arthropoda

compound brain by

fusion of the anterior

cephalic ganglia

separate segmental ganglia

monopartite brain

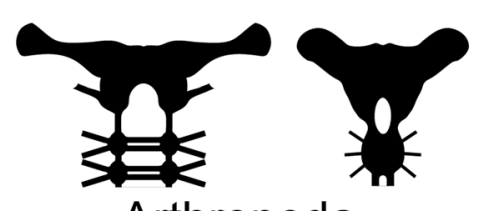


Table 1 Number of fills performed for each commissure and pharyngeal nerve in specimens of Euperipatoides rowelli

\begin{tabular}{ll}
\hline Filled nerve & Number of fills \\
\hline Commissure \#1 & 10 \\
Commissure \#2 & 8 \\
Commissure \#3 & 7 \\
Commissure \#4 & 5 \\
Commissure \#5 & 5 \\
Commissure \#6 & 2 \\
Trunk commissures & 2 \\
Anterior pharyngeal nerve & 3 \\
Posterior pharyngeal nerve & 5 \\
\hline
\end{tabular}

coupled to either tetramethylrhodamine or fluorescein (MW3000, lysine-fixable; Molecular Probes, Eugene, USA) were then added to the well filled with distilled water [43]. The preparations were incubated in the dark for $12-16 \mathrm{~h}$ at $4{ }^{\circ} \mathrm{C}$, after which the well containing dextran was removed and the tissue fixed in $4 \%$ paraformaldehyde in phosphatebuffered saline (PBS; $0.1 \mathrm{M}, \mathrm{pH}$ 7.4) for $2 \mathrm{~h}$ at $4{ }^{\circ} \mathrm{C}$. After several rinses in PBS and dehydration through an ethanol series $(50,70,90,95 \%, 2 \times 100 \%$; 10 min each) the preparations were cleared in methyl salicylate and mounted between two coverslips.

\section{Confocal microscopy, light microscopy and image processing}

Whole-mount preparations of the central nervous system were analyzed with a fluorescence microscope (Leica Leitz DMR; Leica Microsystems, Wetzlar, Germany) and a confocal laser-scanning microscope (Leica TCS STED; Leica Microsystems). Confocal image stacks were processed with Leica ASAF v2.3.5 (Leica Microsystems), IMARIS 7.2.1 (Bitplane, Zurich, Switzerland), and ImageJ $1.48 \mathrm{v}$ (National Institutes of Health, Bethesda, MD, USA). Final panels and diagrams were designed using Adobe (San Jose, CA, USA) Photoshop CS5 and Illustrator CS5. Animations were designed using Adobe Flash CS5.

\section{Additional files}

Additional file 1: Scenario on the evolution of the arthropod brain Animation, swf file. Anterior is up. The compound brain of arthropods (sensu Richter et al. [5]) most likely evolved by a fusion of initially separate proto-, deuto- and tritocerebral ganglia. Note that the tripartite brain of arthropods alternatively might have evolved several times independently, as some groups, including branchiopod crustaceans, show a bipartite brain (e.g. [29]).

Additional file 2: Scenario on the evolution of the onychophoran brain. Animation, swf file. Anterior is up. The composite brain of onychophorans (sensu Richter et al. [5]) most likely evolved by anteriorly moving somata, which give rise to the deutocerebrum.

\section{Authors' contributions}

CM and GM conceived and designed the experiments. CM performed the experiments. CM and GM analyzed the data and wrote the manuscript. Both authors read and approved the final manuscript.

\section{Author details \\ ${ }^{1}$ Animal Evolution and Development, Institute of Biology, University of Leip- zig, Talstraße 33, 04103 Leipzig, Germany. ${ }^{2}$ Department of Zoology, Institute of Biology, University of Kassel, Heinrich-Plett-Str. 40, 34132 Kassel, Germany.}

\section{Acknowledgements}

We are thankful to Noel Tait for organizing the collecting permits, to Paul Sunnucks, David Rowell, Noel Tait, Ivo de Sena Oliveira, Franziska Anni Franke, Sandra Treffkorn, and Michael Gerth for their help with specimen collection and to Vladimir Gross for proofreading the manuscript. The staff members of the State Forests NSW (New South Wales, Australia) are gratefully acknowledged for providing the collecting permits. The Open Access Office of the University of Leipzig is thanked for financial support. This work was supported by a grant from the German Research Foundation (DFG; grant Ma 4147/3-1) to GM, who is a Research Group Leader supported by the Emmy Noether Programme of the DFG.

\section{Compliance with ethical guidelines}

\section{Competing interests}

The authors declare that they have no competing interests.

Received: 19 February 2015 Accepted: 12 August 2015

Published online: 25 August 2015

\section{References}

1. Fanenbruck M, Harzsch S, Wägele JW. The brain of the Remipedia (Crustacea) and an alternative hypothesis on their phylogenetic relationships. Proc Natl Acad Sci USA. 2004;101:3868-73.

2. Homberg U. Neuroarchitecture of the central complex in the brain of the locust Schistocerca gregaria and S. americana as revealed by serotonin immunocytochemistry. J Comp Neurol. 1991;303:245-54.

3. Loesel R, Wolf H, Kenning M, Harzsch S, Sombke A. Architectural principles and evolution of the arthropod central nervous system. In: Minelli A, Boxshall G, Fusco G, editors. Arthropod Biology and Evolution. Heidelberg: Springer; 2013. p. 299-342.

4. Mayer G, Kauschke S, Rüdiger J, Stevenson PA. Neural markers reveal a onesegmented head in tardigrades (water bears). PLoS One. 2013;8:e59090.

5. Richter S, Loesel R, Purschke G, Schmidt-Rhaesa A, Scholtz G, Stach T, et al. Invertebrate neurophylogeny: suggested terms and definitions for a neuroanatomical glossary. Front Zool. 2010;7:29.

6. Scholtz G, Edgecombe GD. The evolution of arthropod heads: reconciling morphological, developmental and palaeontological evidence. Dev Genes Evol. 2006;216:395-415.

7. Mayer G. Onychophora. In: Schmidt-Rhaesa A, Harzsch S, Purschke G, editors. Structure and Evolution of Invertebrate Nervous Systems. Oxford: Oxford University Press; 2015 (in press).

8. Mayer G, Whitington PM, Sunnucks P, Pflüger H-J. A revision of brain composition in Onychophora (velvet worms) suggests that the tritocerebrum evolved in arthropods. BMC Evol Biol. 2010;10:255.

9. Strausfeld NJ, Strausfeld C, Stowe S, Rowell D, Loesel R. The organization and evolutionary implications of neuropils and their neurons in the brain of the onychophoran Euperipatoides rowelli. Arthropod Struct Dev. 2006;35:169-96.

10. Cong P, Ma X, Hou X, Edgecombe GD, Strausfeld NJ. Brain structure resolves the segmental affinity of anomalocaridid appendages. Nature. 2014;513:538-42.

11. Eriksson BJ, Samadi L, Schmid A. The expression pattern of the genes engrailed, pax6, otd and six3 with special respect to head and eye development in Euperipatoides kanangrensis Reid 1996 (Onychophora: Peripatopsidae). Dev Genes Evol. 2013;223:237-46.

12. Franke FA, Mayer G. Controversies surrounding segments and parasegments in Onychophora: insights from the expression patterns of four "segment polarity genes" in the peripatopsid Euperipatoides rowelli. PLoS One. 2014;9:e114383. 
13. Mayer G, Martin C, de Sena Oliveira I, Franke FA, Gross V. Latest anomalocaridid affinities challenged. Nature. 2014;516:E1-2.

14. Martin C, Mayer G. Neuronal tracing of oral nerves in a velvet wormimplications for the evolution of the ecdysozoan brain. Front Neuroanat. 2014;8(7):1-13.

15. Ou Q, Shu D, Mayer G. Cambrian lobopodians and extant onychophorans provide new insights into early cephalization in Panarthropoda. Nat Commun. 2012;3:1261

16. Whitington PM, Mayer $\mathrm{G}$. The origins of the arthropod nervous system: insights from the Onychophora. Arthropod Struct Dev. 2011;40:193-209.

17. Mayer G, Harzsch S. Immunolocalization of serotonin in Onychophora argues against segmental ganglia being an ancestral feature of arthropods. BMC Evol Biol. 2007;7:118.

18. Mayer G, Harzsch S. Distribution of serotonin in the trunk of Metaperipatus blainvillei (Onychophora, Peripatopsidae): implications for the evolution of the nervous system in Arthropoda. J Comp Neurol. 2008;507:1196-208.

19. Mayer G, Whitington PM. Neural development in Onychophora (velvet worms) suggests a step-wise evolution of segmentation in the nervous system of Panarthropoda. Dev Biol. 2009;335:263-75.

20. Henry LM. The nervous system and the segmentation of the head in the Annulata. Microentomology. 1948;13:27-48.

21. von Kennel J. Entwicklungsgeschichte von Peripatus edwardsii Blanch. und Peripatus torquatus n.sp. I. Theil. Arb Zool-Zootom Inst Würzburg. 1885;7:95-229.

22. Boyan GS, Reichert H, Hirth F. Commissure formation in the embryonic insect brain. Arthropod Struct Dev. 2003;32:61-77.

23. Fischer AHL, Scholtz G. Axogenesis in the stomatopod crustacean Gonodactylaceus falcatus (Malacostraca). Invertebr Biol. 2010;129:59-76.

24. Harzsch S. The tritocerebrum of Euarthropoda: a "non-drosophilocentric" perspective. Evol Dev. 2004;6:303-9.

25. Hirth F, Loop T, Egger B, Miller DFB, Kaufman TC, Reichert H. Functional equivalence of Hox gene products in the specification of the tritocerebrum during embryonic brain development of Drosophila. Development. 2001;128:4781-8.

26. Mittmann B, Scholtz G. Development of the nervous system in the "head" of Limulus polyphemus (Chelicerata: Xiphosura): morphological evidence for a correspondence between the segments of the chelicerae and of the (first) antennae of Mandibulata. Dev Genes Evol. 2003;213:9-17.

27. Fedorow B. Zur Anatomie des Nervensystems von Peripatus. I. Das Neurosomit von Peripatus tholloni. Zool Jahrb Abt Anat Ontog Tiere. 1926;48:273-310.

28. Altman JS, Kien J. Functional organization of the subesophageal ganglion in arthropods. In: Gupta AP, editor. Arthropod brain, its evolution, development, structure, and functions. New York: Wiley; 1987. p. 265-301.

29. Kirsch R, Richter $\mathrm{S}$. The nervous system of Leptodora kindtii (Branchiopoda, Cladocera) surveyed with Confocal Scanning Microscopy (CLSM), including general remarks on the branchiopod neuromorphological ground pattern. Arthropod Struct Dev. 2007;36:143-56.

30. Marcus E. Tardigrada. Dr. H. G. Bronns Klassen und Ordnungen des TierReichs wissenschaftlich dargestellt in Wort und Bild. Leipzig: Akademische Verlagsgesellschaft; 1929. p. 1-609.

31. Mayer G, Martin C, Rüdiger J, Kauschke S, Stevenson PA, Poprawa I, et al Selective neuronal staining in tardigrades and onychophorans provides insights into the evolution of segmental ganglia in panarthropods. BMC Evol Biol. 2013;13:230

32. Persson DK, Halberg KA, Jørgensen A, Møbjerg N, Kristensen RM Neuroanatomy of Halobiotus crispae (Eutardigrada: Hypsibiidae): Tardigrade brain structure supports the clade Panarthropoda. J Morphol. 2012;273:1227-45.

33. Schulze C, Neves RC, Schmidt-Rhaesa A. Comparative immunohistochemical investigation on the nervous system of two species of Arthrotardigrada (Heterotardigrada, Tardigrada). Zool Anz. 2014;253:225-35.

34. Zantke J, Wolff C, Scholtz G. Three-dimensional reconstruction of the central nervous system of Macrobiotus hufelandi (Eutardigrada, Parachela): implications for the phylogenetic position of Tardigrada. Zoomorphology. 2008;127:21-36.

35. Gross V, Mayer G. Neural development in the tardigrade Hypsibius dujardini based on anti-acetylated $\alpha$-tubulin immunolabeling. EvoDevo. 2015;6:12.

36. Schürmann FW. Common and special features of the nervous system of Onychophora: a comparison with arthropoda, annelida and some other invertebrates. In: Breidbach O, Kutsch W, editors. The Nervous System of Invertebrates: an evolutionary and comparative approach. Basel: Birkhäuser; 1995. p. 139-58

37. Campbell LI, Rota-Stabelli O, Edgecombe GD, Marchioro T, Longhorn SJ, Telford MJ, et al. MicroRNAs and phylogenomics resolve the relationships of Tardigrada and suggest that velvet worms are the sister group of Arthropoda. Proc Natl Acad Sci USA. 2011;108:15920-4.

38. Dunn CW, Hejnol A, Matus DQ, Pang K, Browne WE, Smith SA, et al. Broad phylogenomic sampling improves resolution of the animal tree of life. Nature. 2008:452:745-9.

39. Rota-Stabelli O, Kayal E, Gleeson D, Daub J, Boore J, Telford M, et al. Ecdysozoan mitogenomics: evidence for a common origin of the legged invertebrates, the Panarthropoda. Genome Biol Evol. 2010;2:425-40.

40. Telford MJ, Bourlat SJ, Economou A, Papillon D, Rota-Stabelli O. The evolution of the Ecdysozoa. Philos Trans R Soc B Biol Sci. 2008:363:1529-37.

41. Baer A, Mayer G. Comparative anatomy of slime glands in Onychophora (velvet worms). J Morphol. 2012;273:1079-88.

42. Robson EA, Lockwood APM, Ralph R. Composition of the blood in Onychophora. Nature. 1966;209:533.

43. Pflüger HJ, Field LH. A locust chordotonal organ coding for proprioceptive and acoustic stimuli. J Comp Physiol A. 1999;184:169-83.

\section{Submit your next manuscript to BioMed Central and take full advantage of:}

- Convenient online submission

- Thorough peer review

- No space constraints or color figure charges

- Immediate publication on acceptance

- Inclusion in PubMed, CAS, Scopus and Google Scholar

- Research which is freely available for redistribution

Submit your manuscript at 